\title{
Cognitive function improvement with astaxanthin and tocotrienol intake: a randomized, double-blind, placebo-controlled study
}

\author{
Takahiro Sekikawa, ${ }^{1, *}$ Yuki Kizawa, ${ }^{1}$ Yanmei Li, ${ }^{2}$ and Tsuyoshi Takara ${ }^{3}$ \\ 1BGG Japan Co., Ltd., 8F Ginza Kobikicho Building, 8-18-1 Ginza, Chuo-ku, Tokyo 104-0061, Japan \\ 2Beijing Gingko-Group Biological Technology Co., Ltd., 1706, Tower A Building 1, Tianzuo Intemationa1 Center, No. 12 Zhongguancun South Avenue, \\ Haidian District, Beijing, China \\ ${ }^{3}$ Medical Corporation Seishinkai, Takara Clinic, 9F Taisei Building, 2-3-2 Higashi-gotanda, Shinagawa-ku, Tokyo 141-0022, Japan
}

(Received 27 November, 2019; Accepted 9 March, 2020; Published online 19 June, 2020)

\begin{abstract}
We examined the effects of the mixed ingestion of astaxanthin derived from Haematococcus pluvialis and tocotrienols on the cognitive function of healthy Japanese adults who feel a memory decline. Forty-four subjects were randomly but equally assigned to the astaxanthin-tocotrienols or placebo group. An astaxanthintocotrienols or placebo capsule was taken once daily before or after breakfast for a 12-week intervention period. The primary outcome was composite memory from the Cognitrax cognitive test, and the secondary outcomes were other cognitive functions and subjective symptoms for memory. Each group included 18 subjects in the efficacy analysis (astaxanthin-tocotrienols group, $55.4 \pm 7.9$ years; placebo group, $54.6 \pm 6.9$ years). The astaxanthintocotrienols group showed a significant improvement in composite memory and verbal memory in Cognitrax at $\Delta 12$ weeks compared with the placebo group. Additionally, the astaxanthin-tocotrienols group showed a significant improvement in the subjective symptom of "During the last week, have you had trouble remembering people's names or the names of things?" compared with the placebo group after 12 weeks. No adverse events were observed in this study. The results demonstrated that taking an astaxanthintocotrienols combination improves the composite memory and verbal memory of Japanese adults who feel a memory decline (UMIN 000031758).
\end{abstract}

Key Words: cognitive, astaxanthin, tocotrienol, Cognitrax, antioxidant properties, lipid peroxidation

$\mathrm{T}$ he damage of nerve cells caused by oxidative stress is one of the factors that decrease cognitive function. ${ }^{(1,2)}$ The accumulation of amyloid $\beta(\mathrm{A} \beta)$ induces oxidative stress and damages tissues around the hippocampal region, leading to the onset of Alzheimer's disease (AD), one of the cognitive impairments. ${ }^{(3)} \mathrm{A} \beta$ is also expressed in healthy people, and the elimination mechanism by the enzyme neprilysin and phagocytes works normally. ${ }^{(4)}$ However, when the elimination function is lowered by aging, $A \beta$ accumulates and the risk of developing AD increases. ${ }^{(5)}$ Moreover, $\mathrm{A} \beta$ begins to accumulate about 20 years before the onset of AD. ${ }^{(6)}$ Therefore, the removal of oxidative stress in the brain at an early stage is effective in the protection of nerve cells, which may suppress the decline in cognitive function as well as prevent and inhibit the progression of AD.

Astaxanthin has antioxidant properties and plays a role in the protection of oxidative damage through a variety of mechanisms, including elimination of singlet oxygen and radicals, suppression of lipid peroxidation, and regulation of gene expression associated with oxidative stress. ${ }^{(7,8)}$ A previous study in vitro has shown that astaxanthin exerts a neuroprotective effect against $\mathrm{A} \beta$ toxicity. ${ }_{.}^{(9)}$ Additionally, clinical research has reported that astaxanthin improves antioxidant capacity ${ }^{(10)}$ and improves antioxidant status in red blood cells. ${ }^{(11)}$ Astaxanthin is specifically absorbed into the blood and can cross the blood-brain barrier in rats, ${ }^{(12)}$ suggesting that it is effective in preventing various disorders caused by reactive oxygen species in brain nerve cells. ${ }^{(13-15)}$ Thus, astaxanthin may suppress cognitive decline.

Astaxanthin on the market has two structures: one is a fatty acid ester, and the other is a non-fatty acid ester. A fatty acid ester comes from Haematococcus pluvialis (H. pluvialis) or krill, whereas a non-fatty acid ester comes from Phaffia rhodozyma or Paracoccus carotinifaciens or is chemically synthesized. ${ }^{(16-18)}$ A previous report in mice showed that the concentrations of astaxanthin derived from $H$. pluvialis in the plasma and liver were higher than those of astaxanthin derived from P. rhodozyma and astaxanthin that was chemically synthesized. ${ }^{(17)}$ This research indicates that, among the sources of astaxanthin, astaxanthin derived from $H$. pluvialis could have high bioavailability.

Tocotrienols are known to cross the blood-brain barrier and reach the brain because of their structure with unsaturated side chains. ${ }^{(19)}$ Furthermore, tocotrienols have neuroprotective effects through their antioxidant effects against oxidative stress induced by $A \beta$ accumulation. ${ }^{(20-22)}$ In studies using aged rats, tocotrienols suppress the decline in memory and learning functions and improve spatial memory with aging. ${ }^{(23,24)}$ Tocotrienols have potent antioxidants with lipoperoxyl radical scavenging. ${ }^{(25)}$ In addition, astaxanthin is highly efficient at inhibiting lipid peroxidation ${ }^{(26)}$ that acts on oxidative damage through a variety of other mechanisms. ${ }^{(7,8)}$ Furthermore, astaxanthin derived from $H$. pluvialis has high bioavailability. ${ }^{(17)}$ The antioxidant capacity of the combination of astaxanthin and tocotrienols is significantly stronger than that of either astaxanthin or tocotrienols alone, because of the hydrogen bonding and intermolecular interactions between these two compounds. ${ }^{(27)}$ Therefore, the simultaneous ingestion of astaxanthin derived from $H$. pluvialis and tocotrienols (AT) may enhance lipid resistance to oxidation more when compared with ingestion of $H$. pluvialis-derived astaxanthin or tocotrienols alone.

To the best of our knowledge, the only evidence on the effects of foods containing the AT combination on human cognitive function was reported by Hongo et al. ${ }^{(28)}$ However, in Hongo et al.'s study, the placebo set was a food product excluding only astaxanthin derived from $H$. pluvialis, and the aim of the study was

To whom correspondence should be addressed.

E-mail: sekikawa@bggjapan.com 
to evaluate the function of astaxanthin derived from $H$. pluvialis. Thus, no functional evaluation was performed when AT was ingested in a mixture. In addition, Hongo et al. ${ }^{(28)}$ confirmed that healthy Japanese adults between 60 and 79 years retained visual information appropriately for a short period and showed a tendency to improve their self-assessment of memory. However, the primary outcome was a multi-item study, indicating included exploratory elements. Therefore, there is still insufficient evidence for the effect of the AT combination on cognitive function. We investigated the effects of the AT combination on cognitive function in healthy Japanese adults aged $\geq 40$ years who feel a memory decline.

\section{Materials and Methods}

Study design. This study was a randomized, double-blind, placebo-controlled study conducted at Medical Corporation Seishinkai, Takara Clinic (Tokyo, Japan), between May 13 and September 1, 2018. The allocation was based on a 1:1 ratio. The study protocol was approved by the independent ethical committee of Medical Corporation Seishinkai, Takara Clinic, on March 13, 2018 (approval no. 1803-1802-BJ01-03-TC). This study was conducted in accordance with the Declaration of Helsinki (2013) and the Ethical Guidelines for Medical and Health Research involving human subjects of Japan and thoroughly considered medical ethics. The protocol was registered at the University Hospital Medical Information Network Clinical Trials Registry (UMIN000031758).

Subjects. Inclusion criteria were defined as follows: (a) experiencing mild forgetfulness in healthy Japanese adult subjects; (b) eligibility to participate in the study by the principal physician; (c) attaining a Mini-Mental Status Examination (MMSE) score of $\geq 24$ at screening/before intake; and (d) relatively lower normalized Cognitrax (Health Solution, Inc., Tokyo, Japan) composite memory domain scores at screening/before intake. Exclusion criteria were defined as follows: (a) a medical history of current treatment for malignancy, heart failure, or myocardial infarction; (b) current treatment for cardiac arrhythmia, hepatic, renal, or cerebrovascular disease, rheumatism, diabetes mellitus, hyperlipidemia, hypertension, or other chronic diseases; (c) a diagnosis of dementia; (d) a diagnosis of mental illnesses such as major depression and attention-deficit hyperactivity disorder; (e) daily consumption of medications (including herbal medicines), "foods for specified health uses," "foods with function claims," or other functional foods/beverages; (f) daily consumption of food containing docosahexaenoic acid, eicosapentaenoic acid, Ginkgo biloba extract, tocotrienol, astaxanthin, $\gamma$-aminobutyric acid, phosphatidylserine, and/or other improved cognitive function foods/beverages; ( $g$ ) allergic reaction to medications and/or products that contain the study ingredients; (h) being pregnant, lactating, or planning to become pregnant; (i) enrollment in other clinical trials within the last 3 months before agreeing to participate in this study; and (j) ineligibility to participate in the study based on the evaluation of the principal physician.

Regularly, all subjects were enrolled through the website (https://www.go106.jp/) operated by ORTHOMEDICO Inc. (Tokyo, Japan) between March 19 and May 12, 2018. The study protocol was comprehensively explained to all the subjects. Written informed consent was obtained from all of them before enrollment in the study at the ORTHOMEDICO Inc. office. Notably, no subject was part of the sponsors or funding companies.

Sample size. Cognitive functions were assessed using Cognitrax based on the CNS Vital Signs (CNS Vital Signs LLC, Morrisville, NC). ${ }^{(29)}$ The primary outcome was an increment in the score of the composite memory domain, which is one of the domains evaluated by Cognitrax. No studies have evaluated the composite memory domain with the intake of AT. Thus, we referred to the study of Gualtieri et al. ${ }^{(29)}$ who used the CNS Vital
Signs, and calculated the SD of the primary outcome. SD in the composite memory domain was calculated to be 7.88 in healthy subjects aged $\geq 20$ years, and we hypothetically obtained a similar $\mathrm{SD}$ in our study. In addition, we hypothesized that a difference in scores of the composite memory domain between the two groups of $\geq 7.00$ points means a clinically significant difference in the improvement of cognitive function. Therefore, the sample size was evaluated with an assumed $\alpha$ value of 0.05 and a $(1-\beta)$ value of 0.80 . Consequently, the sample size was finalized to be 20 subjects per group. Furthermore, we considered $10 \%$ of the dropout rate and added two extra subjects to each group (22 subjects per group).

Enrollment, randomization, and blinding. Of 121 subjects who signed informed consent, eligible subjects who were considered appropriate for the study, attained an MMSE score of $\geq 24$, and did not experience dementia ${ }^{(30)}$ were selected by the physician. In addition, subjects with relatively lower normalized Cognitrax composite memory scores [normalized score evaluated based on the average of scores corresponding to the subjects' age set at 100 $(\mathrm{SD}, 15)]^{(31)}$ before intake were selected as priority subjects for enrollment in this study. An allocation controller equally, but randomly, assigned subjects to either the AT group or the placebo group (P group; $n=22$ per group). The allocation was performed using StatLight \#11 ver. 2.10 (Yukms Co., Ltd., Kawasaki, Japan), a computerized random-number generator. The allocation method was stratified randomization, and the allocation adjustment factor was defined as the normalized Cognitrax composite memory score, sex, and age of screening. Furthermore, subjects, the physician, the assessor of outcomes, and others who were associated with this study were not aware of group assignments and were not involved in the allocation. Moreover, the allocation controller locked the assignment sheet until the key-opening day (October 23, 2018).

Intervention. The test soft capsules included AT (BGG Japan Co., Ltd., Tokyo, Japan; total weight content, $160 \mathrm{mg}$; . pluvialis-derived astaxanthin, approximately $9 \mathrm{mg}$; tocotrienol, approximately $50 \mathrm{mg}$ ) and safflower oil as placebo (total weight content, $160 \mathrm{mg}$ ). All the subjects were asked to consume either one capsule containing AT or one placebo capsule per day before or after breakfast for 12 weeks. Both capsules were declared identical in color, odor, and flavor by the Ethics Committee.

Outcomes. Table 1 outlines the schedule for this study. Subjects visited the clinic and underwent examinations before intake and at 8 and 12 weeks after intake. All subjects abstained from excessive alcohol or exercise from the day before the examination until the end of the examination. Furthermore, they abstained from eating or drinking anything including the test food, except water, for $6 \mathrm{~h}$ before providing blood samples.

(1) Primary outcome: composite memory domain. Cognitrax evaluates various cognitive function domains, such as processing speed, and executive function based on the CNS Vital Signs, ${ }^{(29)}$ evaluating each domain's score from 10 separate tests. The scores of the composite memory domain were calculated as the sum of the verbal memory (VBM) and visual memory (VIM) domain scores. ${ }^{(29)}$ At the beginning of the VBM test, 15 words were presented on the screen, one by one, every $2 \mathrm{~s}$. Next, a subject was asked to identify those words nested among 30 words, including new words (immediate memory scores). Furthermore, the subject was again asked to identify the learned 15 words nested among 30 words, including new words after all the tests had been taken (delayed memory scores). In the VIM test, words in the VBM test were replaced with geometric figures, and the procedure remained the same as that of the VBM test. The composite memory score was calculated from the total number of correct answers given in the VBM and VIM tests and was converted into a normalized score. The normalized value was calculated from the measured value based on a normal distribution with a mean \pm SD of $100 \pm 15$; if the measured value of a subject is $1 \mathrm{SD}$ greater than 
Table 1. Schedule of enrollment, intervention, and assessments

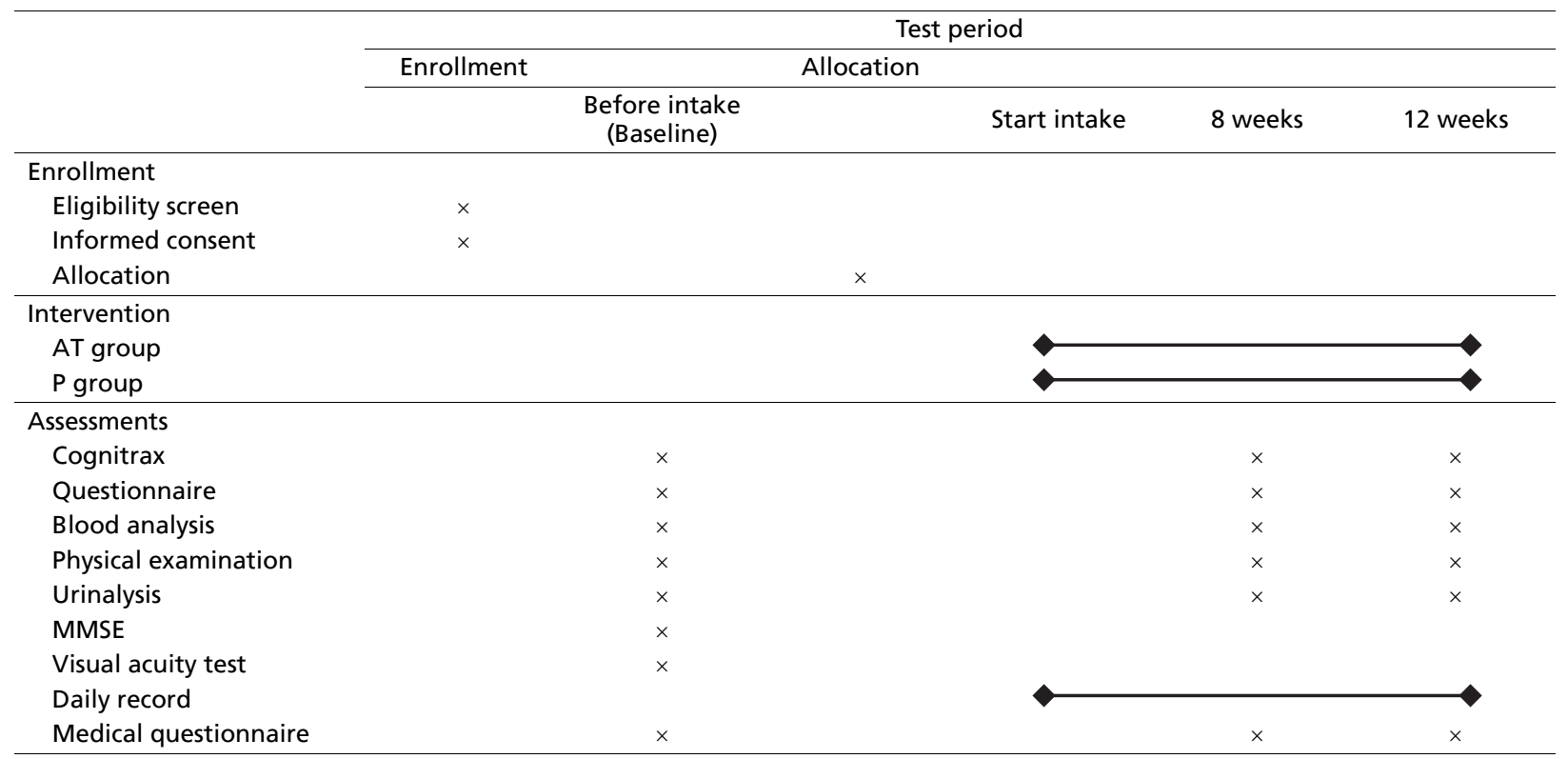

the average of his/her own age, his/her normalized score is 115 . The standard score in domains was assessed as follows: $>109$ points, "Above"; 90-109 points, "Average"; 80-89 points, "Low Average"; 70-79 points, "Low"; and <70 points, "Very Low". (31)

(2) Secondary outcomes. The cognitive functions of the subjects were evaluated using Cognitrax as follows: neurocognitive index domain, VBM domain, VIM domain, psychomotor speed domain, reaction time domain, complex attention domain, cognitive flexibility domain, processing speed domain, executive function domain, social acuity domain, reasoning domain, working memory domain, sustained attention domain, simple attention domain, and motor speed domain.

In addition, subjective symptoms were assessed by the original questionnaire for the Likert scale using the following questions: "Over the past week, have you forgotten things often?", "Have you been concerned about memory loss during the last week?", "Is there any time that you cannot remember a story you heard during the last week?", "During the last week, have you had trouble remembering people's names or the names of things?", "Did you leave behind anything over the last week?", "Did you feel chronically tired during the past week?", "Were you experiencing eye fatigue during the past week?", "Did you experience a stiff neck or shoulders during the past week?", "Have you felt depressed for the past week?", "Did you experience discomfort in your back over the last week?", "Was it difficult to get up from the floor or a chair in the last week?", "Did your knees hurt during crouching or standing up in the last week?", and "Did your knees hurt while going up and down the stairs in the last week?". All these questions were assessed on a scale from 1 (strongly disagree) to 6 (strongly agree).

Subjects' blood samples $(19 \mathrm{ml})$ were collected at the Medical Corporation Seishinkai, Takara Clinic, and tested for the following: brain-derived neurotrophic factor (BDNF), propanoyl lysine (PRL), and pentosidine. In this study, all collected blood samples were entrusted to LSI Medience Corporation (Tokyo, Japan).

(3) Safety assessment. Safety evaluations were assessed in physical examination, urinalysis, and blood analysis (Table 2-4). All subjects were asked to fill out a medical questionnaire to understand their health conditions. In addition, subjects were asked to record a daily report on health conditions, use of medica- tions, and lifestyles

Statistical analysis. All outcomes were assessed before intake and at 8 and 12 weeks after intake. Setting before intake as baseline, each assessment point was subtracted from baseline and reported as the change in the value ( $\Delta 8$ and $\Delta 12$ weeks). In addition, subjects' background and demographic data were aggregated based on age, MMSE, and IgE (radioimmunosorbent test), and data of the AT and P groups were compared using Student's $t$ test.

Cognitive function data at baseline and changes are presented as mean $\pm \mathrm{SD}$, which were analyzed using Student's $t$ test. Moreover, subjective symptoms data were analyzed using the MannWhitney $U$ test at baseline and at 8 and 12 weeks after intake. Physical examination and blood analysis data are presented as mean $\pm \mathrm{SD}$, which were analyzed at baseline using Student's $t$ test. Furthermore, we analyzed data at 8 and 12 weeks after intake using the two-way analysis of covariance (ANCOVA). When ANCOVA was used for data analyses, we used the baseline values as covariates. Of note, between-group comparison was used in the post hoc analysis. Furthermore, urinalysis data were set to a code where 1 was identified as within the normal range and 0 as outside the normal range. The $\chi^{2}$ test was used for between-group analyses.

All statistical analyses in this study were two sided, and we set the significance level to $5 \%$ with no adjustment for multiple comparisons. Data analyses were performed using Windows SPSS ver. 23.0 (IBM Japan, Ltd., Tokyo, Japan).

\section{Results}

Subjects. Figure 1 presents a flowchart of the follow-up of the study subjects. None had an intake rate of less than $90 \%$ throughout the intake period. Four subjects who did not submit a diary or return the test food at the post-trial case review meeting and could not confirm the presence or absence of the intervention and four subjects who were judged as "No" by the validity indicator of Cognitrax were judged as ineligible for analysis and excluded from the analysis. When the breakdown of persons who were excluded from the analysis after the key opening was checked, it was found that two subjects in each group were not able to confirm the presence or absence of the intervention, and 
Table 2. Results of physical examination (AT group, $n=20$; P group, $n=20$ )

\begin{tabular}{|c|c|c|c|c|c|c|c|}
\hline & & Baseline & 8 weeks & 12 meeks & & $p$ value & \\
\hline & & Baseinne & 8 weeks & 12 weeks & Baseline & 8 weeks & 12 weeks \\
\hline Body fat percentage (\%) & $\begin{array}{l}\text { AT group } \\
\text { P group }\end{array}$ & $\begin{array}{l}22.4 \pm 4.1 \\
25.4 \pm 5.6\end{array}$ & $\begin{array}{l}23.1 \pm 3.7 \\
25.4 \pm 5.3\end{array}$ & $\begin{array}{l}23.2 \pm 3.8 \\
25.6 \pm 5.1\end{array}$ & 0.060 & 0.582 & 0.785 \\
\hline $\begin{array}{l}\text { Diastolic blood pressure } \\
(\mathrm{mmHg})\end{array}$ & $\begin{array}{l}\text { AT group } \\
\text { P group }\end{array}$ & $\begin{array}{r}79 \pm 12.6 \\
76.2 \pm 10.7\end{array}$ & $\begin{array}{l}78.5 \pm 11.9 \\
79.3 \pm 12.1\end{array}$ & $\begin{array}{l}78.3 \pm 11.3 \\
78.2 \pm 10.9\end{array}$ & 0.454 & 0.201 & 0.412 \\
\hline Pulse rate (bpm) & $\begin{array}{l}\text { AT group } \\
\text { P group }\end{array}$ & $\begin{array}{l}74.2 \pm 9.3 \\
71.9 \pm 12\end{array}$ & $\begin{array}{l}72.3 \pm 8.9 \\
72.3 \pm 11.2\end{array}$ & $\begin{array}{l}70.6 \pm 9.2 \\
70.1 \pm 8.5\end{array}$ & 0.507 & 0.569 & 0.588 \\
\hline Body temperature $\left({ }^{\circ} \mathrm{C}\right)$ & $\begin{array}{l}\text { AT group } \\
\text { P group }\end{array}$ & $\begin{array}{l}36.1 \pm 0.6 \\
36.1 \pm 0.4\end{array}$ & $\begin{array}{l}36.3 \pm 0.4 \\
36.3 \pm 0.2\end{array}$ & $\begin{array}{l}36.3 \pm 0.4 \\
36.3 \pm 0.3\end{array}$ & 0.948 & 0.932 & 0.550 \\
\hline
\end{tabular}

Data are mean \pm SD.

Table 3. Results of urinalysis

\begin{tabular}{|c|c|c|c|c|c|c|}
\hline & & AT grou & $(n=20)$ & P group & $(n=20)$ & \\
\hline & $\begin{array}{l}\text { Assessment } \\
\text { point }\end{array}$ & $\begin{array}{l}\text { Within } \\
\text { the reference range }\end{array}$ & $\begin{array}{c}\text { Outside } \\
\text { the reference range }\end{array}$ & $\begin{array}{l}\text { Within } \\
\text { the reference range }\end{array}$ & $\begin{array}{c}\text { Outside } \\
\text { the reference range }\end{array}$ & $p$ value \\
\hline & Baseline & 19 & 1 & 20 & 0 & 1.000 \\
\hline Protein & 8 weeks & 18 & 2 & 18 & 2 & 1.000 \\
\hline & 12 weeks & 18 & 2 & 19 & 1 & 1.000 \\
\hline & Baseline & 20 & 0 & 20 & 0 & NA \\
\hline Glucose & 8 weeks & 20 & 0 & 20 & 0 & NA \\
\hline & 12 weeks & 20 & 0 & 20 & 0 & NA \\
\hline & Baseline & 20 & 0 & 20 & 0 & NA \\
\hline Urobilinogen & 8 weeks & 20 & 0 & 20 & 0 & NA \\
\hline & 12 weeks & 20 & 0 & 20 & 0 & NA \\
\hline & Baseline & 20 & 0 & 20 & 0 & NA \\
\hline Bilirubin & 8 weeks & 20 & 0 & 20 & 0 & NA \\
\hline & 12 weeks & 20 & 0 & 20 & 0 & NA \\
\hline & Baseline & 20 & 0 & 20 & 0 & NA \\
\hline $\mathrm{pH}$ & 8 weeks & 20 & 0 & 19 & 1 & 1.000 \\
\hline & 12 weeks & 20 & 0 & 20 & 0 & NA \\
\hline & Baseline & 19 & 1 & 17 & 3 & 0.605 \\
\hline Occult blood & 8 weeks & 19 & 1 & 16 & 4 & 0.342 \\
\hline & 12 weeks & 20 & 0 & 18 & 2 & 0.487 \\
\hline & Baseline & 20 & 0 & 20 & 0 & NA \\
\hline Ketone bodies & 8 weeks & 20 & 0 & 19 & 1 & 1.000 \\
\hline & 12 weeks & 20 & 0 & 20 & 0 & NA \\
\hline
\end{tabular}

Data are the number of subjects. NA, not available.

two subjects in each group were judged as "No" by the validity indicator of Cognitrax. The number of subjects analyzed as the per protocol set was 18 subjects $(8$ men and 10 women, aged $55.4 \pm 7.9$ years) in the AT group and 18 subjects ( 8 men and 10 women, aged $54.6 \pm 6.9$ years) in the $P$ group. The number of subjects analyzed as the safety analysis set was 20 subjects $(9$ men and 11 women, aged $55.3 \pm 7.5$ years) in the AT group and 20 subjects ( 8 men and 12 women, aged $55.4 \pm 7.4$ years) in the $P$ group. The background and age distribution of the study subjects are shown in Table 5-1, 5-2, 5-3, and 5-4. There was no item in which there was any significance between groups in the background factor.

Cognitrax. The results of the cognitive tests are shown in Table 6 and Fig. 2. In composite memory, which was set as the primary outcome, points of $\Delta 12$ weeks in the AT group were significantly higher than those in the $\mathrm{P}$ group (AT group, $20.1 \pm 13.3$ points; $\mathrm{P}$ group, $9.6 \pm 16.2$ points; $p=0.040$; Fig. $2 \mathrm{~A}$ ). The variable points of the VBM domain for $\Delta 12$ weeks were $23.6 \pm 14.2$ points in the AT group and $12.1 \pm 19.0$ points in the $P$ group, which were significantly higher in the AT group than in the $\mathrm{P}$ group ( $p=0.048$; Fig. 2B). In the social acuity domain, the score of the AT group was significantly higher than that of the $\mathrm{P}$ group at baseline (AT group, $97.4 \pm 13.6$ points; $\mathrm{P}$ group, $69.2 \pm 55.3$ points; $p=0.043)$.

Subjective symptoms. The results of the subjective symptoms are shown in Fig. 3. The scale numbers of the question "During the last week, have you had trouble remembering people's names or the names of things?" were median 3.0 
Table 4. Results of blood analysis (AT group, $n=20$; P group, $n=20$ )

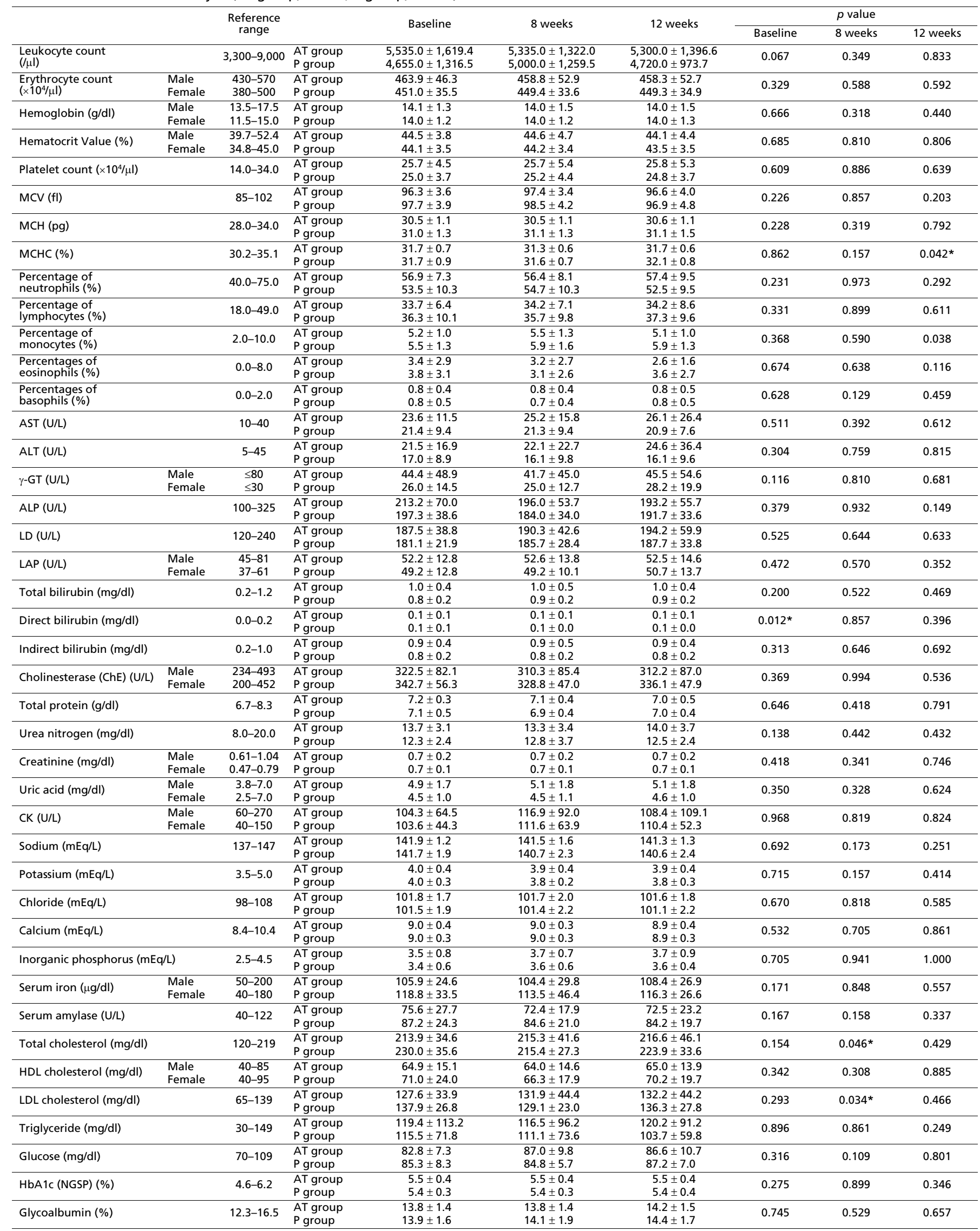

Data are mean $\pm S D .{ }^{*} p<0.05$ vs $P$ group. 


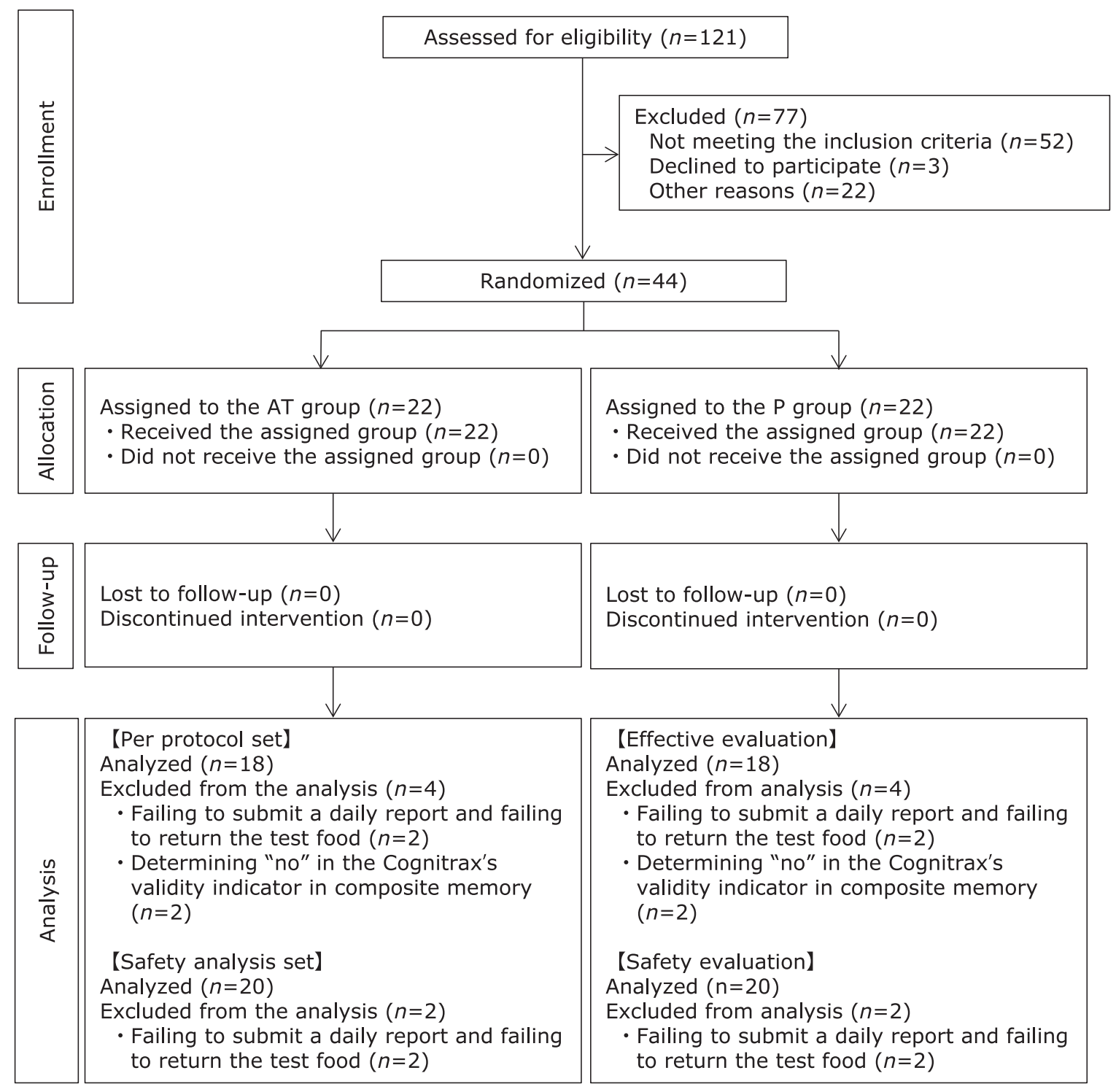

Fig. 1. Flowchart of participants in this study.

Table 5-1. Subjects' background information (per protocol set)

\begin{tabular}{lccc}
\hline & AT group $(n=18)$ & P group $(n=18)$ & $p$ value \\
\hline Age (years) & $55.4 \pm 7.9$ & $54.6 \pm 6.9$ & 0.721 \\
MMSE (points) & $29.2 \pm 1.0$ & $29.4 \pm 0.8$ & 0.373 \\
IgE (RIST) (IU/ml) & $146.5 \pm 181.1$ & $176.1 \pm 244.1$ & 0.683 \\
\hline
\end{tabular}

Data are mean \pm SD.

Table 5-2. Subjects' background information (safety analysis set)

\begin{tabular}{lccc}
\hline & AT group $(n=20)$ & P group $(n=20)$ & $p$ value \\
\hline Age (years) & $55.3 \pm 7.5$ & $55.4 \pm 7.4$ & 0.966 \\
MMSE (points) & $29.0 \pm 1.2$ & $29.2 \pm 1.2$ & 0.604 \\
IgE (RIST) (IU $/ \mathrm{ml})$ & $156.1 \pm 181.2$ & $165.2 \pm 233.9$ & 0.891 \\
\hline
\end{tabular}

Data are mean \pm SD.
Table 5-3. Subjects' age distribution (per protocol set)

\begin{tabular}{lccccc}
\hline \multirow{2}{*}{ Age (years) } & \multicolumn{2}{c}{ AT group $(n=18)$} & & \multicolumn{2}{c}{ P group $(n=18)$} \\
\cline { 2 - 3 } \cline { 5 - 6 } & Men $(n)$ & Women $(n)$ & & Men $(n)$ & Women $(n)$ \\
\hline $40-49$ & 0 & 3 & & 2 & 3 \\
$50-59$ & 5 & 6 & & 5 & 6 \\
$60-69$ & 3 & 0 & & 1 & 0 \\
$\geq 70$ & 0 & 1 & & 0 & 1 \\
\hline
\end{tabular}

Data are the number of subjects.

Table 5-4. Subjects' age distribution (safety analysis set)

\begin{tabular}{lccccc}
\hline \multirow{2}{*}{ Age (years) } & \multicolumn{2}{c}{ AT group $(n=20)$} & & \multicolumn{2}{c}{ P group $(n=20)$} \\
\cline { 2 - 3 } \cline { 5 - 6 } & Men $(n)$ & Women $(n)$ & & Men $(n)$ & Women $(n)$ \\
\hline $40-49$ & 0 & 3 & & 2 & 3 \\
$50-59$ & 6 & 7 & & 5 & 7 \\
$60-69$ & 3 & 0 & & 1 & 0 \\
$\geq 70$ & 0 & 1 & & 0 & 2
\end{tabular}

Data are the number of subjects. 
Table 6. Results of Cognitrax (AT group, $n=18$; P group, $n=18$ )

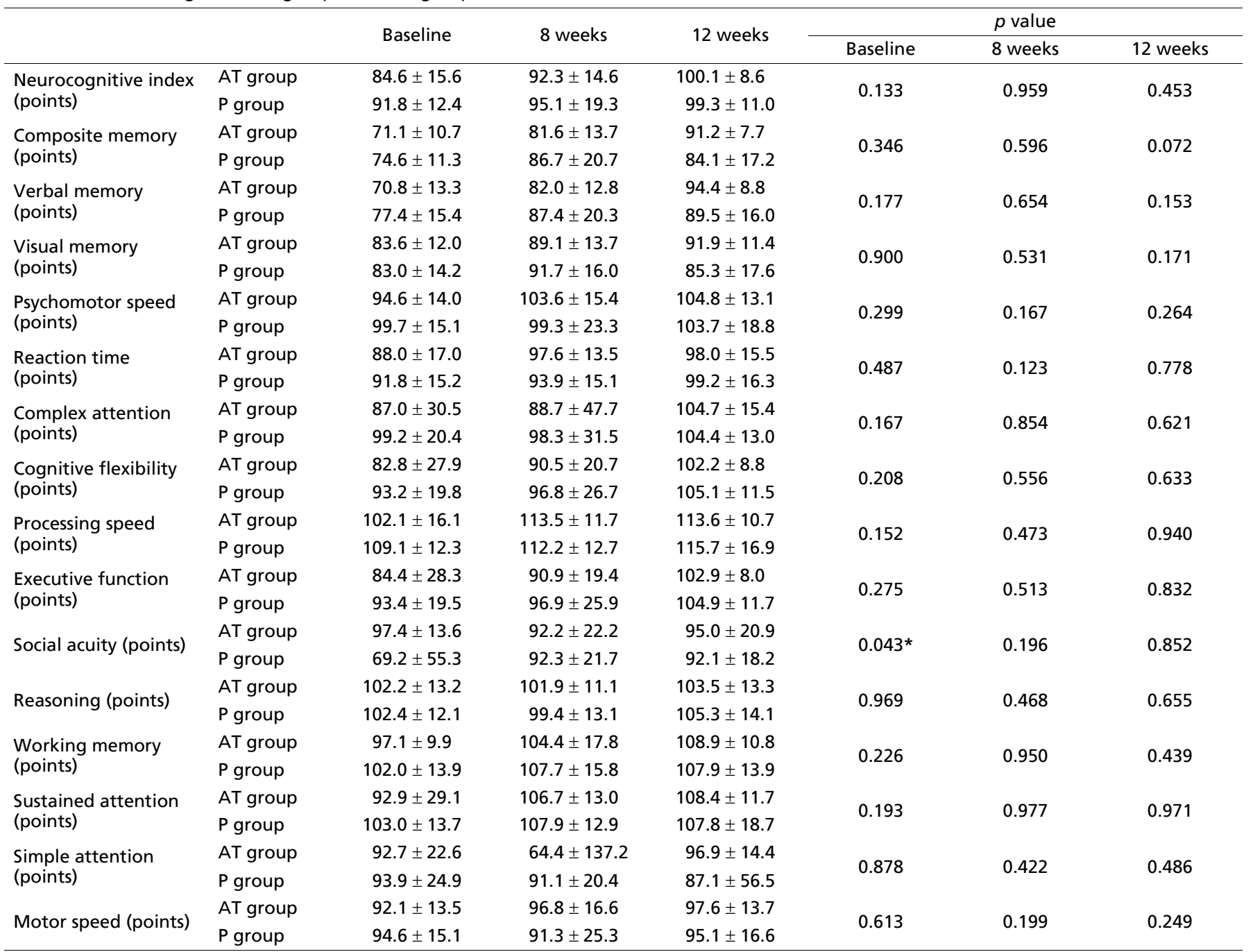

Data are mean \pm SD. ${ }^{*} p<0.05$ vs $\mathrm{P}$ group.

(Q1-Q3, 2.0-3.8) in the AT group and median 4.0 (Q1-Q3, 3.04.8 ) in the $\mathrm{P}$ group at 12 weeks after ingestion, which were significantly lower in the AT group than in the P group $(p=0.036$; Fig. 3). No significant differences were identified for the other questions (data not shown).

Blood BDNF, PRL, and pentosidine levels. There were no significant differences between the groups (data not shown).

Safety assessment. No medically problematic changes were observed with the continued ingestion of the test food.

\section{Discussion}

The purpose of this study was to investigate the cognitive improvement effect of the simultaneous intake of AT $(9 \mathrm{mg}$ /day astaxanthin and $50 \mathrm{mg} /$ day tocotrienol) for 12 weeks by healthy Japanese adults who feel a memory decline.

Cognitrax measures a wide range of cognitive areas, including memory, attention, processing speed, and executive function based on the CNS Vital Signs. ${ }^{(29)}$ The scores for each cognitive area were calculated based on the results of 10 tests. A higher score means a higher cognitive function of the region. According to the report of the CNS Vital Signs, ${ }^{(29)}$ a score of composite memory of healthy subjects peaked in their 20s and then decreased gradually with age. Therefore, we considered that an increasing score of composite memory in Cognitrax was an improvement of cognitive function, setting composite memory as the primary outcome in our study. The variation scores of composite memory and VBM in our study were significantly increased in the AT group compared with those in the $\mathrm{P}$ group at 12 weeks after ingestion. The score of Cognitrax is a standardized score calculated using a mean \pm SD of $100 \pm 15$ for the same age group, with $90-110$ scores considered as "Average" range. ${ }^{(31)}$ Our actual composite memory and VBM scores were less than 90 points (without the range of "Average") at baseline in both groups, but the actual scores at 12 weeks after ingestion were within the range of "Average" only in the AT group. The scores of composite memory and VBM domains in the AT group were significantly increased from before intake to 12 weeks after intake, which allowed the domain scores to fall within the range of "Average," indicating that AT intake improves composite memory and VBM. In Cognitrax, the scores of composite memory are calculated as the sum of the scores of VBM and VIM. ${ }^{(29)}$ Therefore, an improvement in VBM may be involved in the improvement in composite memory.

In the evaluation of subjective symptoms, the scale number of "During the last week, have you had trouble remembering people's names or the names of things?" in the AT group was significantly lower than that in the P group at 12 weeks after inges- 

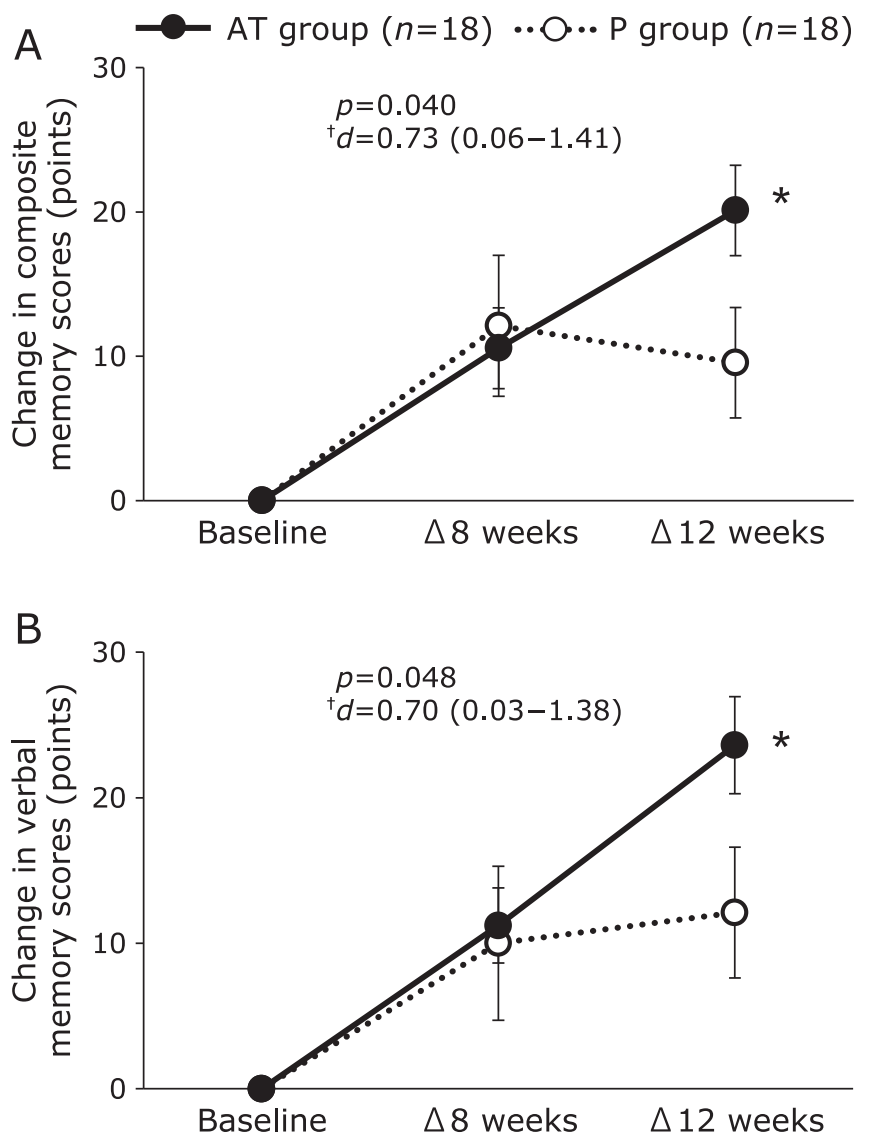

Fig. 2. Change values and actual measured values on the domains in Cognitrax. Data show the change values at baseline and at 8 and 12 weeks after intake ( $\Delta 8$ and $\Delta 12$ weeks): change scores in $(A)$ the composite memory domain and (B) the verbal memory (VBM) domain Closed circle (O), AT group; open circle (O), P group. Data are mean $\pm \mathrm{SE}$. ${ }^{*} p<0.05$ vs $\mathrm{P}$ group. ${ }^{\dagger} \mathrm{Effect}$ size and $95 \%$ confidence interval.

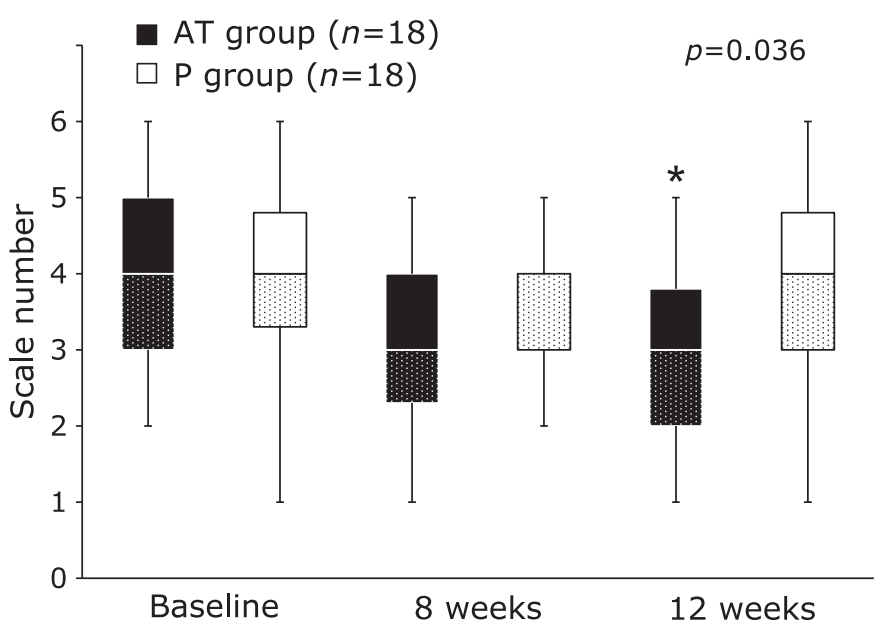

Fig. 3. Results of subjective symptoms. Data show the scale number of "During the last week, have you had trouble remembering people's names or the names of things?" throughout the intervention period. Black bar, AT group; open bar, P group. Data are median (interquartile range). ${ }^{*} p<0.05$ vs $\mathrm{P}$ group. tion. Each item of the original questionnaire means that the lower the scale number, the better the subjective symptoms. Comparing the scale number between the two groups, the median scale number for both groups was 4.0, which means "slightly agree" at the time point before ingestion. However, at 12 weeks after ingestion, the median value for the AT group was 3.0, which means "slightly disagree," whereas the median value for the $\mathrm{P}$ group was 4.0, with no change from pre-ingestion. In other words, the intake of AT increased the number of persons who felt "slightly disagree" in response to the question "During the last week, have you had trouble remembering people's names or the names of things?", which indicates that the intake of AT improved subjective symptoms related to VBM. The results of the objective and subjective evaluations suggested that our results are consistent.

Although no research studies have verified the improvement of cognitive function by a mixed intake of AT, the effectiveness in each component has been reported, including neuroprotection and improvement of cognitive function. Regarding the suppression of cognitive decline because of the neuroprotective effect of astaxanthin, Yook et al.' 's ${ }^{(32)}$ study using mice suggested that ingestion of $H$. pluvialis-derived astaxanthin in the diet at a dose of $0.5 \%$ with mild exercise generated hippocampal neurons and enhanced spatial learning and memory. In addition, Katagiri et al. ${ }^{(33)}$ reported that $H$. pluvialis-derived astaxanthin administered to healthy Japanese adults at a dose of 6 or $12 \mathrm{mg}$ /day was effective on cognitive function. However, Katagiri et al. ${ }^{(33)}$ did not show significant differences between the AT group and the P group. Furthermore, we investigated the effects of $H$. pluvialis-derived astaxanthin taken at $9 \mathrm{mg}$ /day on the cognitive function of healthy Japanese adults, confirming that $H$. pluvialis-derived astaxanthin at $9 \mathrm{mg}$ /day improved both VIM and VBM. ${ }^{(34)}$ In contrast, previous studies of Taridi et al. ${ }^{(23,24)}$ in elderly rats have shown that tocotrienols, another implicated component in our test food, improved memory and learning function and spatial memory with age. Although interaction of the simultaneous intake of AT could not be clarified in this study, it was certain that the simultaneous intake improves composite memory and VBM. Each component has been suggested to have neuroprotective effects to suppress cognitive decline. A previous epidemiologic study in Japanese adults reported that the accumulation of $A \beta$ increased with age and increased rapidly in their $40 \mathrm{~s}^{(35)}$ The average age of subjects in our study was in the $50 \mathrm{~s}$, where damage to neurons had already begun. As these components have inhibitory effects on cognitive function because of their neuroprotective effects, it is possible that each component inhibited neuronal cell damage in our study as well, and it is inferred that AT improved composite memory and VBM.

This study was the first to evaluate the effects of the simultaneous intake of AT on cognitive function. The study clarified that the combined intake of AT improved composite memory and VBM. However, the blood AT levels were not measured. Thus, it was not possible to confirm whether the levels in blood were actually elevated. Astaxanthin is a type of carotenoid, and the decreasing levels of carotenoids, retinol, and tocopherol in the frontal lobe with age are believed to be associated with the pathogenesis of dementia. ${ }^{(36)}$ In addition, epidemiologic studies reported a lower risk of dementia among individuals with high blood tocotrienol levels. ${ }^{(37-39)}$ Therefore, it is important to investigate the relationship between blood AT levels and cognitive function in future studies. Furthermore, the effects of the antioxidant ability of the combined intake of AT on cognitive function could not be confirmed because we did no assess oxidative markers. Thus, the mechanisms by which AT improves cognitive function remain unclear and should be clarified in the future. In other organs, such as the salivary gland, the oxidative stress that accompanies aging has been reported to decrease saliva secretory function in vivo, and the oxidative marker is negatively correlated with the salivary flow rate. ${ }^{(40)}$ Oxidative stress markers may also 
be related to the decrease in cognitive function. Oxidative stress markers will be measured in further studies to investigate the more detailed mechanisms of the effects of AT on cognitive function. Moreover, we did not confirm the participants' level of cognitive function after the decline of AT consumption in this study, and it is still unknown whether the effect of AT is sustainable or not. However, investigation of the sustainable effects after intake of AT is stopped is a task for future studies, with the aim of determining the mechanisms by which AT improves cognitive function.

Safety evaluations were assessed in physical examination, urinalysis, and blood analysis. Although there were several significant differences between the groups, the mean values for all the items were within the reference values, and the physician judged that there was no effect of the test food intake. Therefore, continuous ingestion of the simultaneous intake of AT for 12 weeks was safe under the conditions of this study.

In conclusion, ingestion of AT $(9 \mathrm{mg} /$ day astaxanthin and $50 \mathrm{mg} /$ day tocotrienol) for 12 weeks by Japanese adults who feel a memory decline improves composite memory and VBM. In addition, there were no safety issues under the conditions of this study.

\section{Authors Contributions}

ST, KY, and LY designed the research. TT conducted the research. ST wrote the paper. TT had primary responsibility for the final content. All authors read and approved the final manuscript.

\section{References}

1 Nunomura A, Tamaoki T, Motohashi N, et al. The earliest stage of cognitive impairment in transition from normal aging to Alzheimer disease is marked by prominent RNA oxidation in vulnerable neurons. J Neuropathol Exp Neurol 2012; 71: 233-241.

2 Nunomura A, Castellani RJ, Zhu X, Moreira PI, Perry G, Smith MA. Involvement of oxidative stress in Alzheimer disease. J Neuropathol Exp Neurol 2006; 65: 631-641.

3 Manczak M, Anekonda TS, Henson E, Park BS, Quinn J, Reddy PH. Mitochondria are a direct site of A $\beta$ accumulation in Alzheimer's disease neurons: implications for free radical generation and oxidative damage in disease progression. Hum Mol Genet 2006; 15: 1437-1449.

4 Iwata N, Saido T. Amyloid- $\beta$ peptide metabolism and Alzheimer's disease. Folia Pharmacol Jpn 2003; 122: 5-14. (in Japanese)

5 Takata K. Molecular targeting and translational research for new therapeutic strategies on Alzheimer's disease. Yakugaku Zasshi 2013; 133: 1389-1399. (in Japanese)

6 Jack CR Jr, Knopman DS, Jagust WJ, et al. Hypothetical model of dynamic biomarkers of the Alzheimer's pathological cascade. Lancet Neurol 2010; 9: 119-128.

7 Galasso C, Corinaldesi C, Sansone C. Carotenoids from marine organisms: biological functions and industrial applications. Antioxidants 2017; 6. pii: E96.

8 Dose J, Matsugo S, Yokokawa $\mathrm{H}$, et al. Free radical scavenging and cellular antioxidant properties of astaxanthin. Int J Mol Sci 2016; 17: 103.

9 Chang $\mathrm{CH}$, Chen CY, Chiou JY, Peng RY, Peng CH. Astaxanthine secured apoptotic death of $\mathrm{PC} 12$ cells induced by $\beta$-amyloid peptide 25-35: its molecular action targets. $J$ Med Food 2010; 13: 548-556.

10 Iwabayashi M, Fujioka N, Nomoto K, et al. Efficacy and safety of eightweek treatment with astaxanthin in individuals screened for increased oxidative stress burden. Anti-Aging Med 2009; 6: 15-21.

11 Nakagawa K, Kiko T, Miyazawa T, et al. Antioxidant effect of astaxanthin on phospholipid peroxidation in human erythrocytes. Br J Nutr 2011; 105: 1563-1571.

12 Tso M, Lam T. Method of retarding and amelorating central nervous systemand eye damage. U.S. Patent; 5527533, 1996.

$13 \mathrm{Wu} \mathrm{H}$, Niu H, Shao A, et al. Astaxanthin as a potential neuroprotective agent for neurological diseases. Mar Drugs 2015; 13: 5750-5766.

14 Grimmig B, Kim SH, Nash K, Bickford PC, Douglas Shytle R. Neuroprotec-

\section{Acknowledgments}

The authors thank all the subjects and staff who participated in this study.

\section{Abbreviations}

$\begin{array}{ll}\text { A } \beta & \text { amyloid beta } \\ \text { AD } & \text { Alzheimer's disease } \\ \text { AT } & \text { astaxanthin and tocotrienols } \\ \text { BDNF } & \text { brain-derived neurotrophic factor } \\ \text { H. pluvialis } & \text { Haematococcus pluvialis } \\ \text { MMSE } & \text { Mini-Mental Status Examination } \\ \text { P group } & \text { placebo group } \\ \text { PRL } & \text { propanoyl lysine } \\ \text { VBM } & \text { verbal memory } \\ \text { VIM } & \text { visual memory }\end{array}$

\section{Conflict of Interest}

The sponsor of this study, BGG Japan Co., Ltd., entrusted ORTHOMEDICO, Inc. with conducting the study. ST and KY are a part of BGG Japan Co., Ltd., and LY is a member of Beijing Gingko-Group Biological Technology Co., Ltd. TT (MD) is a part of Medical Corporation Seishinkai, Takara Clinic. TT was the principal investigator and managed the health of the study subjects. tive mechanisms of astaxanthin: a potential therapeutic role in preserving cognitive function in age and neurodegeneration. Geroscience 2017; 39: 19-32.

15 Galasso C, Orefice I, Pellone P, et al. On the neuroprotective role of astaxanthin: new perspectives? Mar Drugs 2018; 16. pii: E247.

16 Sato K, Sugimoto N, Yamada T, Maitani T. Studies on optical isomerism of astaxanthin in natural food colors and principal pigment in phaffia color. Food Hyg Saf Sci (Shokuhin Eiseigaku Zasshi) 2000; 41: 44-47. (in Japanese)

17 Aoi W, Maoka T, Abe R, Fujishita M, Tominaga K. Comparison of the effect of non-esterified and esterified astaxanthins on endurance performance in mice. J Clin Biochem Nutr 2018; 62: 161-166.

18 Hayashi M, Ishibashi T, Maoka T. Effect of astaxanthin-rich extract derived from Paracoccus carotinifaciens on cognitive function in middle-aged and older individuals. J Clin Biochem Nutr 2018; 62: 195-205.

19 Kaneai N, Sumitani K, Fukui K, Koike T, Takatsu H, Urano S. Tocotrienol improves learning and memory deficit of aged rats. J Clin Biochem Nutr 2016; 58: 114-121.

20 Fukui K, Takatsu H, Koike T, Urano S. Hydrogen peroxide induces neurite degeneration: prevention by tocotrienols. Free Radic Res 2011; 45: 681-691.

21 Khanna S, Parinandi NL, Kotha SR, et al. Nanomolar vitamin E alphatocotrienol inhibits glutamate-induced activation of phospholipase A2 and causes neuroprotection. J Neurochem 2010; 112: 1249-1260.

22 Packer L, Weber SU, Rimbach G. Molecular aspects of $\alpha$-tocotrienol antioxidant action and cell signalling. J Nutr 2001; 131: 369S-373S.

23 Taridi NM, Yahaya MF, Teoh SL, et al. Tocotrienol rich fraction (TRF) supplementation protects against oxidative DNA damage and improves cognitive functions in Wistar rats. Clin Ter 2011; 162: 93-98.

24 Taridi NM, Abd Rani N, Abd Latiff A, Ngah WZ, Mazlan M. Tocotrienol rich fraction reverses age-related deficits in spatial learning and memory in aged rats. Lipids 2014; 49: 855-869.

25 Ahsan H, Ahad A, Siddiqui WA. A review of characterization of tocotrienols from plant oils and foods. J Chem Biol 2015; 8: 45-59.

26 Guerin M, Huntley ME, Olaizola M. Haematococcus astaxanthin: applications for human health and nutrition. Trends Biotechnol 2003; 21: 210-216.

27 Kamezaki C, Nakashima A, Yamada A, et al. Synergistic antioxidative effect of astaxanthin and tocotrienol by co-encapsulated in liposomes. $J$ Clin Biochem Nutr 2016; 59: 100-106.

28 Hongo N, Tominaga K, Okuda J, Suzuki M, Miura N. Effects of astaxanthin 
on cognitive function, physical function and mood condition in elderly people: a randomized, double-blind, placebo-control1ed, parallel-group, comparative study. Jpn Pharmacol Ther 2018; 46: 2043-2062.

29 Gualtieri CT, Johnson LG. Reliability and validity of a computerized neurocognitive test battery, CNS Vital Signs. Arch Clin Neuropsychol 2006; 21: 623-643.

30 Folstein MF, Folstein SE, McHugh PR. "Mini-mental state": a practical method for grading the cognitive state of patients for the clinician. J Psychiatr Res 1975; 12: 189-198.

31 CNS Vital Signs ${ }^{\circledR}$ Brief Interpretation Guide. 2017; p. 13. http://www. cnsvs.com/WhitePapers/CNSVS-BriefInterpretationGuide.pdf. Accessed 1 June 2018.

32 Yook JS, Rakwal R, Shibato J, et al. Leptin in hippocampus mediates benefits of mild exercise by an antioxidant on neurogenesis and memory. Proc Natl Acad Sci U S A 2019; 116: 10988-10993.

33 Katagiri M, Satoh A, Tsuji S, Shirasawa T. Effects of astaxanthin-rich Haematococcus pluvialis extract on cognitive function: a randomised, double-blind, placebo-controlled study. J Clin Biochem Nutr 2012; 51: 102107.

34 Sekikawa T, Kizawa Y, Li Y, Takara T. Cognitive function improvement with astaxanthin intake: a randomized, double-blind, placebo-controlled study. Pharmacometrics 2019; 97: 1-13.

35 Morishima-Kawashima M, Oshima N, Ogata H, et al. Effect of apolipoprotein
$E$ allele $\varepsilon 4$ on the initial phase of amyloid $\beta$-protein accumulation in the human brain. Am J Pathol 2000; 157: 2093-2099.

36 Craft NE, Haitema TB, Garnett KM, Fitch KA, Dorey CK. Carotenoid, tocopherol, and retinol concentrations in elderly human brain. J Nutr Health Aging 2004; 8: 156-162.

37 Chin KY, Tay SS. A review on the relationship between tocotrienol and Alzheimer disease. Nutrients 2018; 10. pii: E881.

38 Mangialasche $\mathrm{F}, \mathrm{Xu} \mathrm{W}$, Kivipelto $\mathrm{M}$, et al. Tocopherols and tocotrienols plasma levels are associated with cognitive impairment. Neurobiol Aging 2012; 33: 2282-2290.

39 Mangialasche F, Westman E, Kivipelto M, et al. Classification and prediction of clinical diagnosis of Alzheimer's disease based on MRI and plasma measures of $\alpha$ - $\gamma$-tocotrienols and $\gamma$-tocopherol. J Intern Med 2013; 273: 602 621.

40 Yamauchi Y, Matsuno T, Omata K, Satoh T. Relationship between hyposalivation and oxidative stress in aging mice. J Clin Biochem Nutr 2017; 61: 40 46.

This is an open access article distributed under the terms of the Creative Commons Attribution-NonCommercial-NoDerivatives License (http://creativecommons.org/licenses/by-nc-nd/4.0/). 